\title{
Canadian Policy on Food Allergen Labelling: Consumers' Perspectives Regarding Unmet Needs
}

\author{
Kristin M. Brown ${ }^{1, *}$, Nancy E. Fenton ${ }^{2}$, Larry D. Lynd ${ }^{3}$, Carlo A. Marra ${ }^{3}$, Mark J. Fitzgerald ${ }^{4}$, \\ Stephanie S. Harvard ${ }^{5}$, Meagan Rosenthal ${ }^{6}$, Bonnie Y. L. Chow ${ }^{7}$, Ann E. Clarke ${ }^{8}$, Susan J. Elliott ${ }^{7}$ \\ ${ }^{1}$ School of Public Health and Health Systems, University of Waterloo, Canada \\ ${ }^{2}$ McMaster Institute for Innovation and Excellence in Teaching and Learning, McMaster University, Canada \\ ${ }^{3}$ Faculty of Pharmaceutical Sciences, University of British Columbia, Canada \\ ${ }^{4}$ Vancouver Coastal Health Research Institute, Center for Clinical Epidemiology and Evaluation, Canada \\ ${ }^{5}$ School of Population and Public Health, University of British Columbia, Canada \\ ${ }^{6}$ EPICORE Center, University of Alberta, Edmonton, Canada \\ ${ }^{7}$ Department of Geography and Environmental Management, University of Waterloo, Canada \\ ${ }^{8}$ Faculty of Medicine, University of Calgary, Canada
}

Copyright (C) 2015 Horizon Research Publishing All rights reserved.

\begin{abstract}
It is estimated that $50 \%$ of Canadian households are directly or indirectly affected by food allergies. Since accidental exposure to food allergens can cause life-threatening reactions, the need for accurate allergen labelling is key. In Canada, new food allergen labelling guidelines were mandated in August 2012. While a substantial improvement, gaps remain. This study explored the perspectives of directly affected individuals as well as members of the general public regarding their needs and preferences for precautionary labelling, allergen symbols, and suggestions for improving labelling practices in Canada. Eight qualitative focus groups were conducted with directly affected $(n=27)$ and general public $(n=24)$ consumers in Vancouver, Canada. Focus groups were audio recorded and transcribed verbatim for subsequent thematic analysis using NVivo9. Although "may contain" is recommended by Health Canada, it was the least preferred precautionary statement among research participants. Both participant groups described how many consumers' purchasing habits are indirectly affected by food allergy; hence, their views should be considered in addition to those of directly affected individuals when developing allergen labelling policies. The findings identify future policy needs: 1) mandating and standardizing precautionary/allergen statements and symbols, 2) increased monitoring, and 3) increasing food allergy education and awareness among consumers and manufacturers.
\end{abstract}

Keywords Food Allergy, Food Label; Advisory Labelling, Consumer Preferences

\section{Introduction}

It is estimated that approximately $7 \%$ of the Canadian population has a food allergy [1]. Food allergy is defined as "an adverse health effect arising from a specific immune response that occurs reproducibly upon exposure to a given food" ([2], p. S8). A reaction between IgE antibodies and food protein causes the immune response [3]. This is in contrast to food intolerance, which describes reactions that are not immune-mediated [2]. There is currently no cure for food allergies; hence, allergic individuals must follow strict avoidance diets. Despite avoidance practices, accidental allergen exposure is still possible. Sheth et al.[4] found that $84 \%$ of participants who had been accidentally exposed perceived the cause as either inappropriate labelling of food products, not reading a food label, or ignoring a precautionary statement on a product. Given these accidental exposures can cause life-threatening reactions, the need for improved allergen labelling and consumer label reading is evident.

Recognizing this need, Health Canada amended the Canadian allergen labelling regulations. As of August 2012, manufacturers are required to declare priority allergens (i.e., tree nuts, peanuts, sesame seeds, wheat and triticale, eggs, milk, soybeans, crustaceans, shellfish, fish, and mustard) under specific terms set by Health Canada [5]. Manufacturers may also voluntarily declare priority allergens in a separate "contains" statement directly under the ingredient list. However, precautionary statements warning the consumer about potential cross-contamination (e.g., "may contain [allergen]") remain voluntary as outlined in a 1994 Health Canada policy [6]. Despite the absence of precautionary labelling regulations, Health Canada has since investigated preferences for precautionary statements through an online survey and in-person stakeholder consultations conducted from 2009-2010. Stakeholders (e.g., 
consumers, food industry, research organizations, health professional associations, allergy associations, and government) were asked for their preferences regarding two statements; "may contain [allergen]" was more preferred than "may be present: [allergen]" [7,8]. Health Canada decided to exclude a "not suitable for" statement option because they felt its use was questionable when considering cross-contamination issues. For example, if milk is included in a product's ingredient list but there is also a possibility of peanut cross-contamination, the manufacturer may choose to include a "not suitable for people with peanut allergies" statement. However, the hazard is greater for milk-allergic individuals than peanut-allergic individuals (personal communication, S. La Vieille, February 2014). Resulting from these consultations, in March 2012, Health Canada published recommendations that when manufacturers use precautionary labelling, they should use one allergen statement, "may contain [allergen]". Health Canada also indicated a revised policy would be developed [6].

Outside of Canada, studies have examined the allergen label preferences of directly affected consumers (i.e., food allergic individuals and parents of food allergic children). For example, in England, peanut- and nut-allergic participants preferred voluntary "allergy advice boxes" ([9], p. 972) (i.e., "contains" statements) to ingredient lists. Particularly concerning was that participants wrongly interpreted the absence of allergy advice boxes as the absence of allergens, similar to peanut-free or nut-free statements. When asked for suggestions to improve labels, they requested greater detail and standardization as well as nut-free labelling [9]. Although they believed "may contain" statements were better than no allergen statements, the majority of participants did not find "may contain" helpful, questioning whether it was merely used to protect manufacturers' liability [10]. Greek and Dutch adults who were, or whose children were, allergic to milk, peanuts, tree nuts, or egg expressed frustration regarding precautionary statements as they felt they were overused and limited consumers' food choices [11]. Suggestions for improvement included standardizing ingredient and allergen information, precautionary statements, and allergen symbols.

Comparing surveys of directly affected Americans from 2003 and 2006, researchers found a $10 \%$ decrease in the number of participants that would avoid precautionary statements. For example, participants (parents of food-allergic children) were less likely to avoid, and more likely to purchase, products with precautionary statements, increasing their risk of accidental exposure [12]. Participants were more likely to avoid products with "may contain" than products with statements describing the equipment used or facility. Similarly in an Australian study, $65 \%$ of directly affected parents ignored products with "made in the same factory", while only $22 \%$ ignored "may be present" [13]. However, the majority of participants did not find precautionary statements helpful and expressed confusion regarding their meaning.

Harrington et al. [14] estimated that half of Canadian households are directly or indirectly affected by food allergies. Hence, although the literature has focused on the perspectives of directly affected individuals, members of the general public are often indirectly affected. For example, in Ontario, Sabrina's Law mandates that all school boards have policies and procedures for anaphylactic children [15]. Therefore, many consumers' food choices are indirectly affected by food allergy (e.g., parents required to send allergen-free foods to school with their children and staff working within the school). Other examples include friends and extended family of food allergic individuals and parents whose children's friends are food allergic.

Two quantitative studies have examined the precautionary statement preferences of both directly and indirectly affected individuals. Surveys were used to determine three groups of American consumers' preferences (i.e., food allergic adults, caregivers to food allergic individuals, and adults who did not fit into these categories) [16]. Researchers found that "Allergy Information: May contain peanuts" was the most preferred statement by all three groups. Since no significant differences were found between the groups' preferences for precautionary statements, the authors concluded these groups interpret allergen labels similarly.

Ben-Shoshan et al. [17] used surveys to examine the effect of precautionary statements on the purchasing decisions of directly (i.e., someone in household had food allergies) and indirectly (i.e., no food allergies in household but had purchased or prepared food for someone outside their household with food allergies) affected Canadians. Participants were asked whether they would purchase products with three types of precautionary statements commonly used by Canadian manufacturers:

1) Statements that describe the product (e.g., "may contain");

2) Statements that describe the manufacturing process (e.g., "this product is manufactured in a facility that also manufacturers [allergen]); and,

3) Statements that provide suitability information (e.g., "Not suitable for persons with a [particular allergy]").

Both groups said they would be least likely to purchase products with the "not suitable..." statement.

Few studies have addressed the perceptions of those not directly affected by food allergy (i.e., the general public). The current research expands on the Ben-Shoshan et al. [17] study by using qualitative methods to explore "how" and "why" both directly affected individuals and members of the general public interpret food allergen labels and precautionary statements. Its purpose is to explore the perceptions and preferences of both consumer groups regarding Canadian food allergen labels, specifically related to precautionary labelling, allergen symbols, and location of allergen information, in order to inform future policies.

\section{Materials and Methods}


Eight qualitative focus groups were conducted with two types of consumers: 1) directly affected consumers (i.e., allergic individuals or allergic member(s) in immediate family); and 2) the general public (i.e., no allergic members in immediate family). It is important to note the general public participants may have had others in their lives that were affected by food allergy (e.g., friends, co-workers). Ethical approval from the University of British Columbia Behavioural Research Ethics Board and participant written consent were obtained prior to the discussions. Four focus groups were conducted for each participant group, consisting of 5-9 participants for the first sample group and 5-8 participants for the second sample group.

Participants residing in the City of Vancouver area, British Columbia, Canada, were invited to participate if they were 19 years or older and fluent in both reading and writing English. Recruitment for the directly affected group $(n=27)$ was completed through Anaphylaxis Canada (www.anaphlaxis.org), the largest anaphylaxis support group in Canada. A recruitment email was sent to members enrolled in their database and an advertisement was placed on their website. Members of Anaphylaxis Canada are by definition affected by food allergy either personally or through someone in their household. This information was confirmed at the beginning of the focus groups. The general public sample $(\mathrm{n}=24)$ was recruited through IPSOS Reid, a Vancouver-based public marketing research firm. After screening out those who had allergic individuals in their immediate family, a sample that was representative of the general public was invited to participate. All participants were provided an honorarium of $\$ 50$.

The focus groups explored participant perceptions of current allergen labelling, information needs, and preferences for allergen labels. They were digitally recorded and transcribed verbatim for subsequent thematic analysis using NVivo9. Both deductive and inductive approaches to coding were used to identify themes related to the research objectives and arising from the transcripts [18].

\section{Results}

Participant characteristics for each sample group are provided in Tables 1 and 2. Three main themes emerged from the eight focus groups: 1) preferences for precautionary statements, 2) the use of symbols, and 3) suggestions for improving allergen labelling practices. Similarities and differences between the directly affected and general public participants are highlighted throughout the results.

But first, it is important to note that both the directly affected and general public participants shared the belief that a broad range of consumers is indirectly influenced by food allergies. For example, they discussed friends and extended family that care for, or eat with, food allergic individuals and parents of children who attend school with food allergic children:

"It's my 9-year-old and. . . all the neighbourhood kids in our townhouse complex who only buy granola bars that are peanut-free because they're served with him around. And these 15 kids all play together and every one of those families cares whether this kid is going to be safe or not." (Directly affected)

"I look at the labels because the schools are peanut-free now and they're also, at least my daycare is peanut-free, and I think eggs as well now. So, yeah, I have to check and see what I'm putting in his lunch." (General public)

The stories described indicate that in addition to directly affected individuals, members of the general public are using allergen information on food labels. However, this consumer group is often omitted from consultations regarding allergen labelling.

Table 1. Directly affected participant characteristics ( $\mathrm{n}=27)$.

\begin{tabular}{|c|c|c|}
\hline Characteristic & $\begin{array}{c}\text { Number of } \\
\text { Directly } \\
\text { Affected } \\
\text { Participants } \\
\end{array}$ & $\begin{array}{c}\text { Percent of } \\
\text { Directly } \\
\text { Affected } \\
\text { Participants } \\
\end{array}$ \\
\hline \multicolumn{3}{|l|}{ Age } \\
\hline $19-29$ & 1 & $4 \%$ \\
\hline $30-39$ & 9 & $33 \%$ \\
\hline $40-49$ & 13 & $48 \%$ \\
\hline $50+$ & 4 & $15 \%$ \\
\hline \multicolumn{3}{|l|}{ Gender } \\
\hline Male & 3 & $11 \%$ \\
\hline Female & 24 & $89 \%$ \\
\hline \multicolumn{3}{|l|}{ Average Household Income } \\
\hline$<\$ 50000$ & 4 & $15 \%$ \\
\hline$\$ 51-75000$ & 2 & $7 \%$ \\
\hline$>\$ 75000$ & 19 & $70 \%$ \\
\hline Undeclared & 2 & $7 \%$ \\
\hline \multicolumn{3}{|l|}{ Education Level } \\
\hline High school & 4 & $15 \%$ \\
\hline College & 5 & $19 \%$ \\
\hline Undergraduate & 9 & $33 \%$ \\
\hline Graduate & 9 & $33 \%$ \\
\hline \multicolumn{3}{|l|}{ Allergic Member(s) of Family } \\
\hline Child Only & 14 & $52 \%$ \\
\hline Self Only & 4 & $15 \%$ \\
\hline Self + Child & 4 & $15 \%$ \\
\hline Self + Child + Spouse & 2 & $7 \%$ \\
\hline Child + Spouse & 2 & $7 \%$ \\
\hline Another Member of Family & 1 & $4 \%$ \\
\hline \multicolumn{3}{|l|}{ Allergens of Family Member(s) } \\
\hline Peanut & 23 & $85 \%$ \\
\hline Tree Nut & 19 & $70 \%$ \\
\hline Egg & 8 & $30 \%$ \\
\hline Milk & 6 & $22 \%$ \\
\hline Seafood/Shellfish & 6 & $22 \%$ \\
\hline MSG/Sulphites & 4 & $15 \%$ \\
\hline Soy & 2 & $7 \%$ \\
\hline Kiwi & 2 & $7 \%$ \\
\hline Sunflower & 1 & $4 \%$ \\
\hline Sesame & 1 & $4 \%$ \\
\hline Wheat & 1 & $4 \%$ \\
\hline \multicolumn{3}{|c|}{$\begin{array}{c}\text { At least one of Allergic Family Members has had an } \\
\text { Anaphylactic Reaction }\end{array}$} \\
\hline Yes & 19 & $70 \%$ \\
\hline No & 8 & $30 \%$ \\
\hline
\end{tabular}


Table 2. General public participant characteristics $(n=24)$.

\begin{tabular}{|ccc|}
\hline Characteristic & $\begin{array}{c}\text { Number of } \\
\text { General Public } \\
\text { Participants }\end{array}$ & $\begin{array}{c}\text { Percent of General } \\
\text { Public Participants }\end{array}$ \\
\hline Age & & \\
$19-29$ & 4 & $17 \%$ \\
$30-39$ & 5 & $21 \%$ \\
$40-49$ & 6 & $25 \%$ \\
$50+$ & 7 & $29 \%$ \\
Missing & 2 & $8 \%$ \\
\hline Gender & 12 & \\
Female & 10 & $50 \%$ \\
Male & 2 & $42 \%$ \\
Missing & & $8 \%$ \\
\hline Average Household & & \\
Income & 6 & $25 \%$ \\
$<\$ 40000$ & 8 & $33 \%$ \\
$\$ 40-60000$ & 3 & $13 \%$ \\
$\$ 60-80000$ & 5 & $21 \%$ \\
$>\$ 80000$ & 2 & $8 \%$ \\
Missing & & $46 \%$ \\
\hline Has Children & 11 & $46 \%$ \\
Yes & 11 & $8 \%$ \\
No & 2 & \\
Missing & & \\
\hline & & \\
\hline
\end{tabular}

\subsection{Preferences for Precautionary Statements}

Participants were asked to share their preferences regarding the three types of precautionary statements identified by Ben-Shoshan et al. [17]. The majority of participants in both groups did not find "may contain" statements helpful, describing them as vague because the wording did not explain why the product may contain the allergen:

"Well, the one I hate, which I just saw at the grocery store a couple of days ago was. . ' may contain, blah, blah, blah'. And I find that very ambiguous and sort of wishy-washy." (General public)

“['May contain'] just makes me feel they're gambling on that someone might say, 'Oh, let's give it a shot', or, 'I'll buy it and bring it home to-call the manufacturer', right, whereas just pick one side of the fence. Either just say that you're not going to sell to people that would be at risk, or do something about it so you can." (Directly affected)

Both groups were skeptical that "may contain" was often used for liability concerns:

"Because from my understanding of the phrase 'may contain', it's very vague. And it doesn't give me any concept as to whether there's a real possibility or they just want to cover themselves for liability." (Directly affected)

"...from my point of view, a lot of the labelling is sort of legalistic 'weaselling out', to sort of protect the factory because, you know, it might have touched, as you say, cross-contamination. We're going to say it 'might' contain something." (General public)

With respect to statements describing the manufacturing process, almost all discussion among directly affected participants was positive. They mentioned how detailed information regarding facilities and production (e.g., information regarding the specific production line, "allergen-free" facilities) was most useful because it allowed for more informed decisions:

'I think 'produced in a factory', that's a fact. It's produced in a factory that also produces stuff that's met some peanut. 'Produced on a line, that also produces products with nuts, peanuts, soy', whatever, right, I think that's also very helpful. Because then you know exactly, right. So that is actually, to me, more helpful." (Directly affected)

A few directly affected participants said they would appreciate more detail regarding production in these statements:

“. . .usually, that doesn't tell me if the same machinery's used or what their cleaning practices are like or anything. It's just that it's there in the same plant. It could be on a different line but they still have to say that. And so the contamination might be really low." (Directly affected)

Interestingly, in contrast, the general public participants rarely discussed statements describing the manufacturing process.

The directly affected participants were divided as to whether the third statement (i.e., "not suitable...") was helpful or unhelpful. Those who found it useful argued it simplified their purchasing decisions:

"I think 'not suitable' would be most clear, 'cause then I wouldn't-- I would just 'know', you know, move on. It wouldn't be something I'd think about." (Directly affected)

Others did not think the manufacturer should determine suitability:

"But 'not suitable for a person with', whatever allergy is of no use at all because, you know, I don't think a manufacturer's in a position to judge the severity of someone's allergy or the risk that they may be willing to take." (Directly affected)

However, all mentions of this statement in the general public sample were positive:

"It would be better if it had the third option where it says it's definitely not suitable for someone with a peanut allergy, then you would know for sure, rather than it may or may not." (General public)

In addition, participants in both groups discussed other statements they found helpful because they were clear: "Contains", "Allergen-Free", and "Does Not Contain":

"The nut-free bars. Like, in sort of my situation where we've got a friend visiting, it's nice to see that on the front 'cause otherwise I'm literally reading everything and I'm not $100 \%$ sure whether the chemical is really a form of a nut. . . . So if it says right on the front 'nut-free' or 'made in a nut-free facility', I know it's safe.” (General public) 
Lastly, participants in both groups expressed confusion about the meaning of precautionary statements:

"Does it mean that they don't know where their suppliers-- or what their suppliers are like? If their suppliers have cross-contamination problems? ... Or is [it] that they are manufactured in the same facility? Or the same line?" (Directly affected)

"My daughter has a little friend who is anaphylactic, so we've come into that where if she's over, I have to actually read the labels to see if it has peanuts in it. 'Cause we don't particularly have a peanut allergy, but somebody who comes into the house may. So I do find it somewhat harder to give her certain things when I'm not sure, when it says 'may or may not contain'. It's not really clear." (General public)

\subsection{Allergen Symbols}

The majority of participants in both groups promoted the use of symbols, such as "peanut-free" or "nut-free" logos. Participants discussed how symbols were helpful for all consumers, and especially useful for children and individuals with low English proficiency (i.e., individuals whose first language is not English and those with low literacy levels):

"Everything needs a picture. It's just so much easier to read and understand if there's something to look at, with a visual with it." (General public)

“. . a child of five or whatever who's just learning to read, even can't read, can still share in reading that label. Because that symbol's there and I think early education and, you know, taking responsibility for their allergens is really important. And, yeah, not even ESL [English as a Second Language], there are a lot of people with reading difficulties, you know, and the time factor, anything that can make it easier." (Directly affected)

Additionally, the directly affected group discussed how symbols were useful for consumers who are not directly affected:

"Visuals, if they can do visuals even better, yeah, because that really helps. Not so much us, but other-- like parents that are trying to be helpful and bring the right thing to school." (Directly affected)

Despite their positive perceptions, members of both groups discussed the challenges associated with symbols, specifically that it may be difficult to include a symbol for each potential allergen because there are so many:

"Because if you start breaking it down into ten different symbols, for ten different things, then you will get a convoluted label on a package that is almost indecipherable." (General public)

\subsection{Suggestions for Improving Labelling Practices}

When asked to provide suggestions for improving allergen labels, the general public participants preferred allergen information to be located on the front of packages, while the directly affected preferred it to be near the ingredients:

"So I think it's easier from a consumer point of view to have it sort of labelled on the front, rather than searching through the whole back, turning it over and actually trying to read it. I think people just get frustrated and just don't bother." (General public)

Both groups mentioned labels should be legible and easy to read.

Participants emphasized the importance of regulating food allergen labels, arguing that the government should be responsible for enforcing regulation:

"And they should be given not just the mandate to do it but they should actually be given the ability to enforce that mandate because otherwise these companies are basically going to-- some will comply, some will sort of comply, and some of them will just say, yeah, okay, sure, whatever." (Directly affected)

"It has to be the government because if there's a violation who's going to hold them accountable?" (General public)

When asked whether precautionary statements should be mandatory, all mentions in the directly affected sample and the majority of mentions in the general public sample supported mandating these statements. Secondly, both groups highlighted the need for increased label standardization across brands, including standardizing symbols and the placement of allergen information. The directly affected sample discussed the importance of standardizing the terminology used and emphasized the importance of standardization for general public consumers:

"I think what really needs to happen is more standardization of the labels. What exactly does 'may contain' mean? What exactly does 'processed in a facility that contains', and 'not suitable for' [mean], well, who makes that judgement?" (Directly affected)

"A symbol that could go on packaging on the front. . It could work in the same way that chemicals, like paints and things [symbols] on the trucks work, and that you look in a particular place, if that's an issue that you have." (General public)

When asked whether any products should be exempt from allergen labelling, the general public participants were divided, with some stating that fruits and vegetables should be exempt, while others felt that no products should be excluded. With respect to directly affected participants, there was consensus for the latter argument:

"You have to seek out the ingredients on all these things that are packaged in the store. And, you know, you can buy bulk buns that you stuff in a bag yourself, there [are] no food ingredients anywhere. So I think that those foods should not be exempt." (Directly affected)

In addition to their policy discussion, participants in both groups called for increased education regarding food 
allergen labelling for both manufacturers and consumers:

"If the government would implement education for the manufacturers and food handlers, so they understand why they're required to do these things, like, it would make such a big difference." (Directly affected)

"It's educating the consumer as well. That has to come in to part of it." (General public)

Further, it was evident that even some of the general public participants had limited knowledge regarding food allergies:

"Are there-- what are the secondary-- what are the next most common anaphylactics [sic] out there, besides peanuts?" (General public)

Lastly, both groups discussed the importance of increasing accessibility of allergen information through product websites and helplines:

"I'd like to have a designated person that you can speak to when you are talking about allergies. Because I find you can talk to two different customer service reps and get completely different viewpoints and information." (Directly affected)

"Every product should have a web page with more detailed information that you could do a bit of research on if it's something that mattered to you. And this actually opens up the opportunity for feedback from citizens." (General public)

\section{Discussion}

The findings illustrate how consumers from the general public are often indirectly affected by food allergies; hence, they regularly use allergen labels and their perspectives are important when developing policies regarding food allergen labelling. While the general public preferences for several label aspects were similar to the directly affected group, they differed regarding precautionary statement type and placement of allergen information. Despite representing a small sample, these qualitative findings point to a difference in preferred precautionary statements. This contradicts previous comparisons of these two groups, which have found consensus in precautionary statement preferences $[16,17]$.

While the 2012 changes to food labelling legislation in Canada have gone a long way to help minimize risk and maximize choice for food allergic individuals, participants in this research study indicate there is still work to be done. That is, they addressed a number of topics not included in the 2012 Health Canada food allergen enhanced labelling regulations: 1) precautionary statements; 2) "contains" statements and label placement; 3) symbols, "allergen-free", and "does not contain" statements; 4) enforcement of food allergen labelling; and 5) education.

The majority of participants believed precautionary statements should be mandatory; however, these statements currently remain voluntary. Both the directly affected and general public participants thought "may contain" precautionary statements were the least helpful. This conflicts with Verrill and Choinière's [16] findings that "Allergen Information: May contain" was the most preferred, but aligns with their findings that "may contain" on its own was less preferable. Interestingly, our findings also conflict with those of the Health Canada community stakeholder consultations [6]. Clearly, more research is needed to get a strong sense of how we can protect the health of Canadians while recognizing the needs of manufacturers and policy makers.

The two groups differed in their preferences for an alternative precautionary statement. While the directly affected group favoured statements that described the manufacturing process, the general public preferred the "not suitable" statement. These preferences reflect how these consumer groups use labels differently: the general public participants preferred statements that clearly recommend whether or not the product should be purchased, simplifying their decisions. In contrast, the directly affected consumers preferred statements that support making purchasing decisions at their own discretion. The findings for the general public align with the Ben-Shoshan et al. [17] study, where both indirectly and directly affected participants viewed "not suitable" as the most effective precautionary statement. However, the current study's directly affected group was divided on the helpfulness of "not suitable", differing from the Ben-Shoshan et al. [17] findings. These results support further research in order to explore the best alternative to "may contain" statements.

Under the current Health Canada regulations, "contains" statements that identify priority allergens in a product (separate from the ingredient list) are voluntary. Based on participant feedback, it would be valuable for the general public if these statements were made mandatory, so they can quickly recognize allergens without reading the entire label. This change, of course, would require significant consultation with the manufacturing sector, as well as other major stakeholders (e.g., distributors and consumers). Additionally, although the current regulations state (voluntary) "contains" statements should be placed with the ingredients, the majority of general public participants preferred this information to be located on the front of packages. Similarly, the Health Canada stakeholder consultations [8] and Voordouw et al. [11] found that participants preferred symbols to be located on the front of packages. Further research should be conducted to determine whether these views are held in a broader sample.

Thirdly, symbols, "allergen-free", and "does not contain" statements are not included in the current regulations; however, they were perceived as useful for members of the general public, children, and people with low English proficiency. Standardization of allergen symbols was emphasized by both groups, aligning with previous research $[9,11]$. Hence, these label components should be included in stakeholder consultations regarding future policy 
considerations.

Consistent with the findings from the Health Canada consultations with consumers and allergy associations [8], participants emphasized the importance of enforcement to ensure that manufacturers are meeting the Health Canada guidelines. Although the Health Canada consultations found that food industry stakeholders preferred voluntary inclusion of precautionary statements [8], this study's results indicate that consumers may need something more. Enforcement should be addressed in future policies in order to hold manufacturers accountable for meeting policy guidelines and improve consumers' purchasing experiences. Both groups emphasized educating manufacturers and consumers about food allergy and allergen labelling practices, aligning with recommendations in the literature $[4,9]$.

Lastly, this research is not without limitations; the directly affected sample was primarily recruited through Anaphylaxis Canada, which may have led to selection bias, as membership in support groups is associated with higher income and education. It is important to address these issues further in a larger cohort of both directly and indirectly affected consumer groups.

\section{Conclusions}

While the 2012 Health Canada food allergen labelling regulations have substantially improved food allergen labels for allergic consumers, the participants in this research reported that there remain areas for improvement; for example,

1) Mandating and standardizing precautionary and "contains" statements across manufacturers;

2) Introducing requirements for the use and standardization of symbols, "allergen-free", and "does not contain" statements;

3) Increased monitoring of precautionary statements; and,

4) Increasing education to promote awareness of food allergies and allergen labelling among manufacturers and consumers.

Of course, addressing all of these aspects will require a multisectoral, multistakeholder process and much negotiation and pilot testing. Although these recommendations apply to the Canadian policy environment, they are likely also applicable to allergen labelling policies worldwide, as these areas are not currently addressed in other countries' policies [19]. This is underscored by a recent position paper that reviewed food allergen risk assessment and management recommendations for the food industry [20].

It is also important to consider the needs of various consumer groups using allergen labels (including directly and indirectly affected individuals, children, and the general public) when developing policies related to food allergen labelling. Children, indirectly affected individuals, and members of the general public are less knowledgeable about food allergens than food allergic adults and parents of food allergic children. Therefore, they have different needs; specifically, allergen information must be easily accessible for them. By increasing education to indirectly affected consumers, these individuals can become advocates and educate other members of the general public. Considering the strong influence of food labels on accidental exposure to allergens, it is important that the needs of all consumer groups are reflected in food allergen labelling policies. Increased education and improved allergen labelling will ultimately lead to safer environments for both directly and indirectly affected and general public consumers, as well as a more level playing field for the food industry. The results of this small, exploratory study will be fed into a large national survey to be conducted in Canada in 2015, in order to ground truth these findings.

\section{Acknowledgements}

This research was funded by Allergy, Genes, and Environment (AllerGen) Network of Centres of Excellence. The writing team is sincerely grateful to Laurie Harada, Executive Director, Anaphylaxis Canada, for support and help with recruitment for this research.

\section{REFERENCES}

[1] L. Soller, M. Ben-Shoshan, D. W. Harrington, J. Fragapane, L. Joseph, Y. St Pierre, S. B. Godefroy, S. La Vieille, S. J. Elliott, A. E. Clarke. Overall prevalence of self-reported food allergy in Canada. Journal of Allergy and Clinical Immunology. Vol.130, No.4, 986-988, 2012.

[2] J.A. Boyce, A. Assa'ad, J. W. Burks, S. M. Jones, H. A. Sampson, R. A. Wood, M. Plaut, S. F. Cooper, M. J. Fenton, S. H. Arshad, S. L. Bahna, L. A. Beck, C. Byrd-Bredbenner, C. A. Camargo Jr, L. Eichenfield, G. T. Furuta, J. M. Hanifin, C. Jones, M. Kraft, B. D. Levy, P. Lieberman, S. Luccioli, K. M. McCall, L. C. Schneider, R. A. Simon, F. E. Simons, S. J. Teach, B. P. Yawn, J. M. Schwaninger. Guidelines for the diagnosis and management of food allergy in the United States: Report of the NIAID-sponsored expert panel. Journal of Allergy and Clinical Immunology. Vol.126, No. 6S, S1-S58, 2010.

[3] A. Gelincik, S. Büyüköztürk, H. Gül, E. Işık, H. İşsever, F. Özşeker, B. Çolakoğlu, M. Dal, Ö. Ayvaz, G. Güngör, A. Akkor. Confirmed prevalence of food allergy and non-allergic food hypersensitivity in a Mediterranean population. Clinical \& Experimental Allergy. Vol.38, No. 8, 1333-1341, 2008.

[4] S. S. Sheth, S. Waserman, R. Kagan, R. Alizadehfar, M. Primeau, S. Elliot, Y. St. Pierre, Wickett, R., R. Joseph, L. Harada, Dufresne, C., Allen, M., Allen, M., S. B. Godefroy, A. E. Clarke. Role of food labels in accidental exposures in food-allergic individuals in Canada. Annuals of Allergy, Asthma \& Immunology. Vol.104, No.1, 60-65, 2010.

[5] Government of Canada. Regulations amending the food and drug regulations $(1220$ - Enhanced labelling for food 
allergen and gluten sources and added sulphites), Online available from

http://gazette.gc.ca/rp-pr/p2/2011/2011-02-16/html/sor-dors2 8-eng.html

[6] Health Canada. The use of food allergen precautionary statements on prepackaged foods, Online available from http://www.hc-sc.gc.ca/fn-an/alt_formats/pdf/label-etiquet/al lergen/precaution_label-etiquette-eng.pdf

[7] Health Canada. Consultation document on precautionary labelling of priority allergens in prepackaged foods, Online available from

http://www.hc-sc.gc.ca/fn-an/consult/_allergen2009/draft-eb auche-eng.php\#Table1

[8] Health Canada. Consultation on Health Canada's food allergen precautionary labelling policy renewal - Summary of comments from

the online and face-to-face consultations, Online available from http://www.hc-sc.gc.ca/fn-an/consult/_allergen2009/labellin g-consult-etiquetage-eng.php

[9] J. Barnett, J. Leftwich, K. Muncer, K. Grimshaw, R. Shepherd, M. M. Raats, M. H. Gowland, J. S. Lucas. How do peanut and nut-allergic consumers use information on the packaging to avoid allergens? Allergy Vol.66, No.7, 969-978, 2011.

[10] J. Barnett, K. Muncer, J. Leftwich, R. Shepherd, M. Raats, M. H. Gowland, K. Grimshaw, J. S. Lucas. Using 'may contain' labelling to inform food choice: a qualitative study of nut allergic consumers. BMC Public Health, Vol.11, p.734, 2011.

[11] J. Voordouw, J. R. Cornelisse-Vermaat, V. Yiakoumaki, G. Theodoridis, G. Chryssochoidis, L. J. Frewer. Food allergic consumers' preferences for labelling practices: a qualitative study in a real shopping environment. International Journal of Consumer Studies, Vol.33, No.1, 94-102, 2009.

[12] S.L. Hefle, T. J. Furlong, L. Niemann, H. Lemon-Mule, S. Sicherer, S. L. Taylor. Consumer attitudes and risks associated with packaged foods having advisory labeling regarding the presence of peanuts. Journal of Allergy and
Clinical Immunology,Vol.120, No.1, 171-176, 2007.

[13] G. Zurzolo, J. Koplin, M. Mathai, T. Mimi, K. Allen. Perceptions of precautionary labelling among parents of children with food allergy and anaphylaxis. The Medical Journal of Australia. Vol.198, No.11, 621-623, 2013.

[14] D.W. Harrington, S. J. Elliott, A. E. Clarke, M. Ben-Shoshan, S. Godefroy. Exploring the determinants of the perceived risk of food allergies in Canada. Human and Ecological Risk Assessment: An International Journal, Vol.18, No.6, 1338-1358, 2012.

[15] Government of Ontario. Sabrina's Law, 2005, Online available

fromhttp://www.e-laws.gov.on.ca/html/statutes/english/elaw s_statutes_05s07_e.htm

[16] L. Verrill, C. J. Choinière. Are food allergen advisory statements really warnings? Variation in consumer preferences and consumption decisions. Journal of Food Products Marketing, Vol.15, No.2, 139-151, 2009.

[17] M. Ben-Shoshan, S. Sheth, D. Harrington, L. Soller, J. Fragapane, L. Joseph, Y. St Pierre, S. La Vielle, S. Elliott, S. Waserman, R. Alizadehfar, L. Harada, M. Allen. M. H. Allen, A. E. Clarke. Effect of precautionary statements on the purchasing practices of Canadians directly and indirectly affected by food allergies. Journal of Allergy and Clinical Immunology. Vol.129, No.5, 1401-1404, 2012.

[18] B. F. Crabtree, W. L. Miller. Doing Qualitative Research 2nd Ed., Sage Publications, Inc, Thousand Oaks, CA, 1999.

[19] S. M. Gendel. Comparison of international food allergen labeling regulations. Regulatory Toxicology and Pharmacology. Vol.63, No.2, 279-285, 2012.

[20] A. Muraro, K. Hoffmann - Sommergruber, T. Holzhauser, L. Poulsen, M. Gowland, C. Akdis, E. N. C. Mills, N. Papadopoulos, G. Roberts, S. Schnadt, R. van Ree, A. Sheikh, S. Vieths. EAACI Food Allergy and Anaphylaxis Guidelines. Protecting consumers with food allergies: understanding food consumption, meeting regulations and identifying unmet needs. Allergy, 2014. 